Quim. Nova, Vol. 33, No. 1, 220-224, 2010

\title{
O VALOR ENERGÉTICO DOS ALIMENTOS. EXEMPLO DE UMA DETERMINAÇÃO EXPERIMENTAL, USANDO CALORIMETRIA DE COMBUSTÃO
}

\author{
Rui C. Santos \\ Centro de Química e Bioquímica, Faculdade de Ciências, Universidade de Lisboa, 1649-016 Lisboa, Portugal
}

Recebido em 25/11/08; aceito em 10/6/09; publicado na web em 13/11/09

\begin{abstract}
FOOD ENERGY VALUES. EXAMPLE OF AN EXPERIMENTAL DETERMINATION, USING COMBUSTION CALORIMETRY. The energies involved in the combustion, under atmosphere of oxygen, of breakfast cereals and dehydrate powdered whole milk samples, were determined by combustion calorimetry. This practical work, in the field of human nutrition, involved the characterization of the nutritional composition and the combustion of samples of the two foods that are part of the alimentary diet, namely, at breakfast. The obtained results allowed to assess the energy value printed in the foods labels and discuss the way those values are estimated.
\end{abstract}

Keywords: energy value; Atwater method; nutrition.

\section{INTRODUÇÃO}

Na indústria alimentar, o valor energético dos alimentos é maioritariamente determinado através da aplicação de um método empírico introduzido no final do século XIX por Atwater. ${ }^{1,2}$ Este método, conhecido como Regra 4-9-4, baseia-se na composição nutricional dos alimentos. As quantidades dos nutrientes (em gramas) maioritariamente usados pelo corpo humano para a obtenção de energia (proteínas, lípidos e glícidos) são multiplicadas, respectivamente, pelos factores 4, 9 e 4 ( $\left(\mathrm{em} \mathrm{kcal.g} \mathrm{g}^{-1}\right)$, para a obtenção do valor energético do alimento.

Embora este método de estimativa possua algumas desvantagens, ${ }^{3-5}$ tais como basear-se no conceito de energia metabolizável, ${ }^{6}$ não fazer distinção entre os vários tipos de glícidos e proteínas, não contabilizar o valor energético das fibras alimentares fermentáveis (solúveis) e não funcionar correctamente para bebidas alcoólicas, ainda é actualmente usado pela indústria alimentar. Nos Estados Unidos da América ${ }^{7,8}$ e na União Europeia, ${ }^{9,10}$ os métodos oficiais de estimativa do valor energético dos alimentos baseiam-se no método descrito, com alguns refinamentos adicionais ao nível da utilização de factores mais específicos para os vários tipos de nutrientes, de acordo com as suas origens.

$\mathrm{Na}$ maior parte dos casos, um alimento não possui uma composição química que se possa exprimir com exactidão por uma fórmula molecular definida. Por este facto, a energia libertada durante o processo de combustão de um alimento no interior de um calorímetro é designada por Poder Calorífico. O valor energético de um alimento pode ser determinado num calorímetro de combustão a partir do valor do Poder Calorífico Superior (PCS) a volume constante, ou seja, através da energia libertada na reacção de oxidação total sob atmosfera de oxigénio puro à pressão de 1 bar $(0,1 \mathrm{MPa})$ e à temperatura de referência de 298,15 K. ${ }^{11} \mathrm{O}$ valor do PCS assim obtido considera que toda a água no final da combustão se encontra na fase líquida. Esta determinação vai resultar na obtenção de um valor energético do alimento maior que o fornecido ao corpo humano, devido à não contabilização das perdas de energia metabólica e de azoto no organismo. De facto, tanto no corpo humano como num calorímetro de combustão, os lípidos e os glícidos, que são constituídos principalmente por carbono, oxigénio e hidrogénio, podem ser oxidados até aos seus produtos finais: dióxido de carbono e água. No entanto, as proteínas também contêm azoto e este elemento, juntamente com

\footnotetext{
*e-mail: rjcs@fc.ul.pt
}

algum carbono e hidrogénio, é expelido pelo organismo humano principalmente sob a forma de ureia. Por este motivo, as proteínas são oxidadas incompletamente no organismo humano, enquanto o são totalmente num calorímetro. A menor eficiência energética do metabolismo humano também tem outras causas: os constituintes produtores de energia não são completamente hidrolisados ou digeridos no corpo humano; e os produtos da digestão não são completamente absorvidos. Assim, para a estimativa do valor energético de um alimento é necessário ter-se em conta a eficiência da sua utilização pelo corpo humano, a qual é representada pelo coeficiente de digestibilidade $^{2}$ do alimento. $\mathrm{O}$ valor deste coeficiente é calculado de acordo com o processo descrito na literatura, ${ }^{12}$ utilizando os coeficientes de digestibilidade individuais e a energia mássica de combustão de cada um dos nutrientes (proteínas, lípidos e glícidos) para cada tipo de alimento compilados por Merrill e Watt, ${ }^{2}$ e a percentagem de cada um dos nutrientes presentes no alimento. Os coeficientes utilizados para as proteínas têm que ser previamente corrigidos através da subtracção do factor $1,25 \mathrm{kcal} . \mathrm{g}^{-1}\left(5,23 \mathrm{~kJ}^{\mathrm{g}} \mathrm{g}^{-1}\right)$ de proteína presente no alimento, valor assumido como médio para contabilizar a perda de azoto incompletamente oxidado pelo organismo humano e libertado na urina. ${ }^{2}$ Contudo, este valor proposto por Atwater não é totalmente aceite por todos os investigadores nesta área. ${ }^{13,14}$ Para a obtenção do valor energético experimental de um alimento ou nutriente, o valor de $P C S$ determinado experimentalmente tem que ser multiplicado pelo respectivo coeficiente de digestibilidade.

No âmbito de uma disciplina sobre nutrição e alimentação, montou-se um trabalho laboratorial para testar a aplicabilidade da Regra 4-9-4 e procedeu-se à determinação experimental dos PCS de uma amostra de um cereal de pequeno-almoço e de leite gordo em pó. Os respectivos valores de PCS foram corrigidos através da contabilização do azoto incompletamente oxidado e da aplicação dos respectivos coeficientes de digestibilidade, sendo os valores energéticos obtidos comparados com os valores estimados através da aplicação da Regra 4-9-4 à composição nutricional fornecida nos rótulos das embalagens dos alimentos.

\section{PARTE EXPERIMENTAL}

\section{Materiais}

Utilizou-se uma amostra de cereais Corn Flakes ${ }^{\circledR}$ (flocos de milho tostado) da marca Kellogg ${ }^{\circledR}{ }^{\circledast} .{ }^{15}$ A composição qualitativa da amostra 
constante no rótulo da embalagem é: arroz, açúcar, extracto de malte, sal, niacina, ferro, vitamina B6, riboflavina (B2), tiamina (B1), ácido fólico, vitamina $\mathrm{D}$ e vitamina $\mathrm{B} 12$. A respectiva informação nutricional é apresentada na Tabela 1. Como informação relevante, o fabricante indica que o produto possui, apenas, $1 \%$ de gordura.

Tabela 1. Informação nutricional apresentada no rótulo da embalagem dos cereais Kellogg's Corn Flakes ${ }^{\circledR}$

\begin{tabular}{|c|c|c|}
\hline & \multicolumn{2}{|c|}{ Por $100 \mathrm{~g}$} \\
\hline Valor energético & \multicolumn{2}{|c|}{$1.600 \mathrm{~kJ} / 370 \mathrm{kcal}$} \\
\hline Proteínas & \multicolumn{2}{|c|}{$6 \mathrm{~g}$} \\
\hline Hidratos de carbono, dos quais: & \multicolumn{2}{|c|}{$85 \mathrm{~g}$} \\
\hline Açúcares & \multicolumn{2}{|c|}{$7 \mathrm{~g}$} \\
\hline Amido & \multicolumn{2}{|c|}{$78 \mathrm{~g}$} \\
\hline Lípidos, dos quais: & \multicolumn{2}{|c|}{$0,8 \mathrm{~g}$} \\
\hline Saturados & \multicolumn{2}{|c|}{$0,2 \mathrm{~g}$} \\
\hline Colesterol & \multicolumn{2}{|c|}{$0 \mathrm{mg}$} \\
\hline Fibras alimentares & \multicolumn{2}{|c|}{$2,5 \mathrm{~g}$} \\
\hline Sódio & \multicolumn{2}{|c|}{$0,9 \mathrm{~g}$} \\
\hline Humidade máx. & \multicolumn{2}{|c|}{$8 \%$} \\
\hline Vitaminas e minerais: & & $\% \mathrm{DDR}$ \\
\hline Niacina & $15 \mathrm{mg}$ & 85 \\
\hline Vitamina B6 & $1,7 \mathrm{mg}$ & 85 \\
\hline Riboflavina (B2) & $1,3 \mathrm{mg}$ & 85 \\
\hline Tiamina (B1) & $1,2 \mathrm{mg}$ & 85 \\
\hline Ácido fólico & $167 \mu \mathrm{g}$ & 85 \\
\hline Vitamina D & $4,2 \mu \mathrm{g}$ & 85 \\
\hline Vitamina B12 & $0,8 \mu \mathrm{g}$ & 85 \\
\hline Ferro & $7,9 \mathrm{mg}$ & 55 \\
\hline
\end{tabular}

DDR - Dose Diária Recomendada pela União Europeia.

Os procedimentos recomendados para secar amostras de alimentos variam de autor para autor. ${ }^{16} \mathrm{O}$ teor em água de um alimento está relacionado com o seu valor nutritivo, pois quanto maior for a quantidade de água presente menor será a quantidade dos constituintes orgânicos com carácter energético para o organismo humano. No caso da amostra de cereal optou-se por proceder à secagem durante 7 h a 383,15 K numa estufa com circulação de ar. Após a secagem, a amostra foi arrefecida num exsicador e triturada num moinho para café. A análise elementar conduziu aos seguintes valores médios (2 ensaios independentes): $\mathrm{C}, 43,34 \% ; \mathrm{H}, 6,85 \% ; \mathrm{N}, 1,38 \% ; \mathrm{S}<0,3 \%$. O teor médio de cinzas obtido foi de $3,14 \%$ (3 ensaios independentes) e o teor médio de humidade foi de $1,16 \%$ ( 3 ensaios independentes).

Utilizou-se uma amostra de leite gordo em pó instantâneo NIDO da marca Nestle $e^{\circledast} \cdot{ }^{17}$ A composição da amostra segundo o rótulo da embalagem é: leite inteiro, emulsionante (lecitina de soja $0,2 \%$ ) e vitaminas. A respectiva informação nutricional apresentada na Tabela 2 indica que o teor de matéria gorda do produto é de $26,2 \%$.

A análise elementar a esta amostra conduziu aos seguintes valores médios (2 ensaios independentes): C, 50,05\%; H, 8,46\%; N, 4,35\%; $\mathrm{S}<0,3 \%$. O teor médio de cinzas obtido foi de $6,32 \%$ (3 ensaios independentes) e o teor médio de humidade foi de $3,10 \%$ (3 ensaios independentes).

Utilizou-se ácido benzoico $\left(\mathrm{C}_{7} \mathrm{H}_{6} \mathrm{O}_{2}\right.$, Parr, padrão termoquímico $)$ para a determinação do equivalente energético do calorímetro.
Tabela 2. Informação nutricional apresentada no rótulo da embalagem do leite gordo em pó Nestlé $N I D O^{\circledR}$

\begin{tabular}{|c|c|c|}
\hline & \multicolumn{2}{|c|}{ Valores médios por $100 \mathrm{~g}$} \\
\hline Valor energético & \multicolumn{2}{|c|}{$2.073 \mathrm{~kJ} / 496 \mathrm{kcal}$} \\
\hline Proteínas & \multicolumn{2}{|c|}{$26,4 \mathrm{~g}$} \\
\hline Glícidos & \multicolumn{2}{|c|}{$38,6 \mathrm{~g}$} \\
\hline Lípidos & \multicolumn{2}{|c|}{$26,2 \mathrm{~g}$} \\
\hline Vitaminas e minerais: & & $\%$ DDR \\
\hline Vitamina A (enriquecido) & $900 \mu \mathrm{g}$ & 112 \\
\hline Vitamina C & $10 \mathrm{mg}$ & 16 \\
\hline Vitamina D (enriquecido) & $8 \mu \mathrm{g}$ & 160 \\
\hline Vitamina E (enriquecido) & $4 \mathrm{mg}$ & 40 \\
\hline Tiamina (B1) & $0,2 \mathrm{mg}$ & \\
\hline Riboflavina (B2) & $1,4 \mathrm{mg}$ & 81 \\
\hline Niacina & $1,0 \mathrm{mg}$ & \\
\hline Vitamina B6 & $0,2 \mathrm{mg}$ & \\
\hline Ácido pantoténico (B5) & $2,0 \mathrm{mg}$ & 30 \\
\hline Vitamina B12 & $1,8 \mu \mathrm{g}$ & 180 \\
\hline Cálcio & $930 \mathrm{mg}$ & 116 \\
\hline Fósforo & $750 \mathrm{mg}$ & 94 \\
\hline Ferro & $1 \mathrm{mg}$ & \\
\hline
\end{tabular}

DDR - Dose Diária Recomendada de vitaminas e minerais segundo directiva da União Europeia.

Com o objectivo de tornar tanto quanto possível igual a elevação de temperatura dos ensaios de calibração com os das amostras de alimentos, e também auxiliar a ignição das amostras, adicionou-se em cada ensaio uma quantidade adequada de $n$-hexadecano $\left(\mathrm{C}_{16} \mathrm{H}_{34}\right.$, Merck, 99\%). A energia específica de combustão padrão desta substância foi previamente determinada no sistema calorimétrico.

No interior da bomba e para a lavagem da mesma após os ensaios, foi utilizada água destilada e desionizada obtida através de um sistema MiliQ $50^{\circledR}$ (Millipore Corporation, condutividade $\leq 0,1 \mu \mathrm{S} . \mathrm{cm}^{-1}$ ). A solução de hidróxido de sódio $0,01 \mathrm{~mol} \cdot \mathrm{dm}^{-3}$ utilizada como titulante foi preparada a partir da diluição de uma ampola de solução padrão de hidróxido de sódio 0,1 mol.dm ${ }^{-3}$ (Merck, Titrisol ${ }^{\circledR}$ ).

\section{Equipamento}

Nos ensaios calorimétricos foi utilizado um calorímetro adiabático de bomba de oxigénio Parr Instrument Co, modelo $1241 .{ }^{18} \mathrm{O}$ seu sistema original de aquisição de temperaturas foi modificado. Este equipamento é recomendado para massas de amostras de aproximadamente $1 \mathrm{~g}$ ou que libertem até um máximo de $33,5 \mathrm{~kJ}$ por ensaio. Optou-se por colocar o calorímetro a funcionar em modo isoperibol devido a ser o modo de operação mais simples de executar. ${ }^{19}$

A aquisição dos valores de temperatura foi realizada através da interface RS-232C de um computador com um programa de aquisição e recolha de dados de resistência, medidos através de um multímetro digital (Agilent Technologies, modelo 34401A), provenientes de uma sonda termistor (Parr, modelo 105E, resistência nominal: $2,2 \mathrm{M} \Omega$ a $T$ = 298,15 K). Esta sonda está imersa na água do vaso calorimétrico, permitindo a medição de resistências com uma precisão de $\pm 10^{-4} \Omega$ na escala de $10 \mathrm{M} \Omega$ (o que equivale a medir temperaturas com uma resolução de $\left.10^{-6} \mathrm{~K}\right)$. 


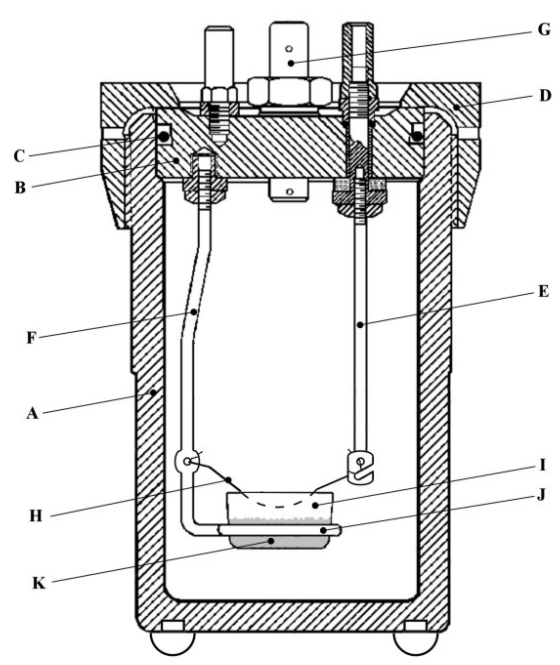

Figura 1. Desenho esquemático da bomba de combustão utilizada. A, corpo da bomba; B, cabeça da bomba; $C$, anel de viton; $D$, anel com rosca; $E$, eléctrodo isolado da cabeça da bomba; $F$, eléctrodo suporte ligado à cabeça da bomba; $G$, válvula de entrada do oxigénio; H, fio fusível; I, cadinho em aço inox; J, anel suporte do cadinho; $K$, amostra

A bomba de combustão utilizada (Parr, modelo 1108, Figura 1) consiste num vaso de pressão em aço inox com um volume interno de $342 \mathrm{~cm}^{3}$, constituído por uma cabeça e um corpo que ficam solidários através de um anel roscado.

A ignição das amostras é obtida pela descarga manual de $22,8 \mathrm{~V}$ (AC) de um condensador através de um fio fusível em liga de níquelcrómio (Parr, modelo 45C10) de 0,16 mm de diâmetro.

O cadinho utilizado (Parr, modelo 43AS) é feito em aço inox (25 $\mathrm{mm}$ de diâmetro x 1,0 mm de espessura x $13 \mathrm{~mm}$ de profundidade), tendo previamente sido aquecido numa mufla durante $4 \mathrm{~h}$ a 753,15 $\mathrm{K}$, de modo a desenvolver um filme protector de óxido em toda a superfície.

As amostras de ácido benzoico e de leite gordo em pó foram comprimidas em pastilhas com cerca de 11,0 mm de diâmetro, com o auxílio de uma prensa (Parr, modelo 2811). No caso das amostras de cereal optou-se por colocar a amostra em pó no fundo do cadinho por ser difícil a sua agregação.

As amostras dos alimentos, o cadinho e o auxiliar de combustão ( $n$-hexadecano) foram pesados com uma exactidão de $\pm 10^{-4} \mathrm{~g}$ numa balança analítica mecânica (Bosch, modelo S2000), enquanto o vaso calorimétrico e a água foram pesados com uma exactidão de $\pm 1 \mathrm{~g}$ numa balança técnica electrónica (Mettler, modelo PE 24). Todas as massas aparentes ao ar medidas neste trabalho foram corrigidas para o vazio ${ }^{20}$ antes de serem utilizadas nos cálculos.

As análises elementares foram efectuadas num aparelho da marca Fisons Instruments, modelo EA1108. O erro estimado para estas análises é $\pm 0,3 \%$ no $\mathrm{C}$ e $\pm 0,1 \%$ no $\mathrm{H}, \mathrm{N}$ e S. Para as determinações do teor de cinza total dos alimentos ( $4 \mathrm{~h}$ a $823,15 \mathrm{~K}$ ), utilizou-se uma mufla da marca Naber Industrieofenbau (modelo L51/S), de acordo com o procedimento descrito na NP $872 .{ }^{21} \mathrm{O}$ teor em humidade foi determinado colocando os alimentos numa estufa durante $6 \mathrm{~h}$ a 380,15 $\mathrm{K}$ até peso constante, tal como descrito na NP $875 .{ }^{22}$

\section{Procedimento experimental}

Colocam-se $10 \mathrm{~cm}$ de fio fusível entre os dois eléctrodos situados na cabeça da bomba. O peso individual do cadinho, da pastilha (ou do pó) da amostra e, quando necessário, do auxiliar de combustão é registado com exactidão de $\pm 10^{-4} \mathrm{~g}$. Adiciona-se $(1,00 \pm 0,01) \mathrm{mL}$ de água destilada e desionizada ao fundo da bomba de combustão. ${ }^{23}$ A cabeça da bomba, já com o cadinho e a amostra a ensaiar no seu interior, é colocada no respectivo corpo, ajustando-se o conjunto com auxílio de um anel roscado. Purga-se o interior da bomba com oxigénio para eliminar o azoto atmosférico e carrega-se a bomba com 30 atm (3,04 MPa) de oxigénio.

Pesa-se o vaso calorimétrico vazio e adicionam-se $(2.000 \pm 1) \mathrm{g}$ de água destilada. Em seguida, o conjunto vaso calorimétrico mais água é colocado dentro do banho termostático e coloca-se a bomba no interior do vaso calorimétrico.

A tampa do calorímetro é fechada e o conteúdo do vaso calorimétrico (bomba+água) é aquecido até próximo da temperatura pretendida para o início do ensaio. Após a estabilização da temperatura da água do vaso, procede-se à recolha dos valores de resistência lidos pelo multímetro em intervalos de $2 \mathrm{~s}$. Passados $900 \mathrm{~s}$ procede-se ao disparo manual do sistema de ignição do calorímetro, ocorrendo a combustão da amostra com o correspondente aumento da temperatura da água do vaso calorimétrico. A recolha dos valores de resistência do sistema é deixada durante mais $1.800 \mathrm{~s}$, até se alcançar o equilíbrio e o ensaio poder ser considerado terminado.

O sistema calorimétrico é desligado e a bomba é retirada do vaso calorimétrico. A válvula de saída de gases é aberta lentamente, a bomba desmontada e o seu interior é lavado com água para um Erlenmeyer para posterior determinação do ácido nítrico formado, ${ }^{24}$ por titulação com uma solução de hidróxido de sódio $0,01 \mathrm{~mol}_{\mathrm{dm}}{ }^{-3}$, usando vermelho de metilo como indicador. O comprimento dos pedaços de fio fusível não queimados é medido para se contabilizar a energia envolvida na combustão do fio fusível (energia de ignição). O cadinho é lavado, calcinado e guardado em exsicador para futura utilização.

De acordo com a recomendação termoquímica, devem-se realizar pelo menos cinco determinações para a obtenção da energia de combustão de uma amostra.

\section{RESULTADOS E DISCUSSÃO}

Pelo facto de existirem trocas de energia entre o vaso calorimétrico e o banho termostático durante os ensaios de combustão (modo isoperibol), as elevações de temperatura observadas necessitam de ser corrigidas para condições adiabáticas através do método de Regnault-Pfaundler. ${ }^{11,25}$

O equivalente energético do calorímetro, $\varepsilon^{\circ}$, foi calculado através da combustão de pastilhas de ácido benzoico com massas próximas de 1,2 g, de acordo com o procedimento experimental já descrito. Estas determinações conduziram a um valor médio $\left\langle\varepsilon^{\circ}\right\rangle=(8.763,50$ $\pm 18,63) \mathrm{J} . \mathrm{K}^{-1}$, onde a incerteza apresentada corresponde ao desvio padrão da média. Esta incerteza corresponde a $0,21 \%$ de $\left\langle\varepsilon^{\circ}\right\rangle$, valor similar ao do limite superior recomendado pela Norma ISO 1928, ${ }^{11}$ que é $0,20 \%$.

Como anteriormente se referiu, foi necessário utilizar um auxiliar de combustão ( $n$-hexadecano). Os resultados obtidos para a energia específica de combustão padrão do $n$-hexadecano conduziram ao valor médio $\left\langle\Delta_{\mathrm{c}} u^{\circ}\right\rangle\left(n-\mathrm{C}_{16} \mathrm{H}_{34}\right)=-(47.294,0 \pm 6,6) \mathrm{J} \cdot \mathrm{g}^{-1}$. A incerteza indicada corresponde ao desvio padrão da média dos resultados experimentais.

\section{Poder calorífico superior (PCS) das amostras de alimentos}

As energias de combustão das amostras foram obtidas numa bomba calorimétrica a volume constante, de modo que as variações de energia observadas correspondem a variações de energia interna. Designa-se por Energia Bruta a quantidade de energia libertada por unidade de massa (a volume constante) quando uma amostra sofre 
combustão em excesso de oxigénio à pressão de 1 bar $(0,1 \mathrm{MPa})$ e à temperatura de 298,15 K. Após a combustão, os produtos da reacção são, para além do oxigénio remanescente, o dióxido de carbono, o dióxido de enxofre e o azoto no estado gasoso, bem como a água líquida em equilíbrio com o seu vapor. Usualmente, para além destes componentes, forma-se ainda um resíduo (cinza) resultante da matéria mineral contida no alimento. $\mathrm{O}$ dióxido de enxofre produzido na reacção (caso a amostra contenha enxofre), tal como os óxidos de azoto resultantes da reacção do azoto atmosférico, considerado contaminante da atmosfera gasosa da bomba, são recolhidos no final de cada ensaio sob a forma de ácidos sulfúrico e nítrico aquosos.

Experimentalmente os valores de $P C S$ são determinados numa bomba calorimétrica a uma pressão de 3,04 MPa e não de $0,1 \mathrm{MPa}$, e a uma temperatura próxima de $298 \mathrm{~K}$. As correcções (designadas por correcções de Washburn ${ }^{25}$ ) que se têm que aplicar aos resultados experimentais, para corrigir as diferenças de pressão e temperatura experimentais para a pressão padrão $(0,1 \mathrm{MPa})$ e para a temperatura de referência de $298,15 \mathrm{~K}$, são desprezáveis quando comparadas com os valores de $P C S$ obtidos experimentalmente e os respectivos erros médios associados, no caso de amostras de alimentos. Convém também salientar que as referidas correcções de Washburn não são facilmente estimadas para os alimentos do presente estudo, dado que um alimento não possui uma composição química que se possa exprimir por uma fórmula molecular definida. Assim, a aplicação deste tipo de correcções é normalmente ignorada neste tipo de determinações na área alimentar. Contudo, nos casos dos ensaios de calibração com ácido benzoico e nos de determinação do calor de combustão da substância auxiliar utilizada ( $n$-hexadecano), tais correcções foram efectuadas e os valores apresentados anteriormente encontram-se corrigidos para as condições padrão de pressão e temperatura.

O PCS a volume constante dos alimentos ensaiados neste trabalho foi calculado através da equação: ${ }^{11}$

$P C S / \mathrm{Jg} .{ }^{-1}=\frac{Q_{\text {totalr. }}-Q_{\text {sec. }}}{m(\text { alim })}$

A energia total produzida durante a reacção de combustão do alimento, $Q_{\text {total }}$, é dada por:

$Q_{\text {total }} / \mathrm{J}=\varepsilon^{\circ} \cdot \Delta T_{\mathrm{ad}}$

onde $\varepsilon^{\circ}$ é o equivalente energético do calorímetro (previamente determinado) e $\Delta T_{\text {ad }}$ a elevação de temperatura adiabática obtida pela combustão da massa de alimento, $m$ (alim), dentro da bomba. Na Equação $1, Q_{\text {r.sec. }}$ representa a contribuição de todas as reacções secundárias que têm lugar durante o processo de combustão, tais como: a combustão do fio fusível; a formação de ácido nítrico aquoso; a variação de energia associada à combustão da substância auxiliar, que no caso destes ensaios correspondeu ao $n$-hexadecano; e, caso existisse enxofre em quantidade mensurável nas amostras dos alimentos, a decomposição do ácido sulfúrico aquoso formado.

$\mathrm{O}$ valor médio de $P C S$ obtido para cada uma das amostras de alimento ensaiado foi: $\langle P C S\rangle($ cereal $)=(16.898,7 \pm 71,3){\mathrm{J} . \mathrm{g}^{-1} \mathrm{e}}$
$<P C S\rangle$ (leite $)=(22.576,3 \pm 38,0) \mathrm{J} \cdot \mathrm{g}^{-1}$. As incertezas apresentadas correspondem aos desvios padrão das médias dos resultados experimentais.

Para estimar os valores energéticos destes alimentos, é necessário considerar-se a eficiência da absorção de energia pelo corpo humano. Assim, o PCS de cada alimento tem de ser multiplicado pelo respectivo coeficiente de digestibilidade. ${ }^{2}$ Aplicando o método mencionado anteriormente, ${ }^{12}$ estimaram-se para o cereal e para o leite em pó, respectivamente, coeficientes de digestibilidade de 0,95 e 0,91 , conduzindo aos seguintes valores energéticos: $(1.605,4 \pm 7,1)$ $\mathrm{kJ} / 100 \mathrm{~g}$ para o cereal; e $(2.054,4 \pm 3,8) \mathrm{kJ} / 100 \mathrm{~g}$ para o leite em pó.

De modo a ilustrar a magnitude dos erros que podem resultar de operações deficientes numa determinação do PCS de um alimento com o sistema calorimétrico utilizado, tomou-se como exemplo um dos ensaios com a amostra de cereal (ver Tabela 3).

Tabela 3. Exemplo da magnitude dos erros experimentais que podem resultar de operações deficientes numa determinação do poder calorífico superior $(P C S)$ com a amostra de cereal ensaiada

\begin{tabular}{lc}
\hline Erro experimental & $\begin{array}{c}\text { Consequência no valor do } P C S \text { em } \\
\mathrm{J} \mathrm{g}^{-1} \text { de amostra de cereal }\end{array}$ \\
\hline 1 mL na titulação do ácido & $\pm 0,60$ \\
$1 \mathrm{~cm}$ na medição da quantidade & $\pm 9,60$ \\
de fio fusível queimado & $\pm 14,99$ \\
1 g no peso da água & $\pm 16,95$ \\
1 mg no peso da amostra & $\pm 17,48$ \\
0,002 K na medição da eleva- &
\end{tabular}

Caso todos os erros referidos pesassem no mesmo sentido

Erro total de 59,61

O método utilizado para a determinação do valor energético dos alimentos impresso nos rótulos das embalagens nunca é referido pelos fabricantes. Na Tabela 4, os valores energéticos impressos nas embalagens dos alimentos ensaiados são comparados com aqueles obtidos através da utilização da Regra 4-9-4 e pelo método calorimétrico descrito neste trabalho. No caso dos cereais Kellogg's Corn Flakes $^{\circledR}$, o valor apresentado em calorias pode ser reproduzido utilizando a Regra 4-9-4. Comparado com o valor energético obtido experimentalmente (Tabela 4), verifica-se uma boa concordância entre os dois valores. Para o leite gordo em pó Nestlé NIDO ${ }^{\circledR}$, o valor apresentado no rótulo em calorias (e em joules) também deve ter sido estimado aplicando a Regra 4-9-4. No caso deste alimento, o valor energético estimado é também semelhante ao valor determinado de modo experimental (ver Tabela 4)

Ressalve-se que os valores energéticos apresentados nos rótulos das embalagens podem ser arredondados para o valor inteiro mais próximo, ou mesmo para outros valores (múltiplos de 5 ou $10 \mathrm{kcal}$ ). Este procedimento é permitido, por exemplo, pela lei da rotulagem de alimentos dos Estados Unidos da América. ${ }^{8}$

Tabela 4. Comparação dos valores energéticos das amostras de alimentos ensaiados

\begin{tabular}{|c|c|c|c|c|c|}
\hline \multirow{2}{*}{ Amostra } & \multicolumn{5}{|c|}{ Valor energético (em kJ/100 g e kcal/100 g) } \\
\hline & Embalagem & Regra 4-9-4 & Desvio (\%) & Experimental & Desvio $(\%)$ \\
\hline \multirow{2}{*}{ Cereais Kellogg's Corn Flakes ${ }^{\circledast}$} & 1.600 & $1.553,1$ & 2,9 & $1.605,4 \pm 7,1$ & 0,3 \\
\hline & 370 & 371,2 & 0,3 & $383,7 \pm 1,7$ & 3,7 \\
\hline \multirow{2}{*}{ Leite Gordo em Pó Nestlé NIDO ${ }^{\circledast}$} & 2.073 & $2.074,4$ & 0,1 & $2.054,4 \pm 3,8$ & 0,9 \\
\hline & 496 & 495,8 & 0,0 & $491,0 \pm 0,9$ & 1,0 \\
\hline
\end{tabular}




\section{CONCLUSÕES}

Os resultados obtidos indicam que o equipamento calorimétrico e o procedimento experimental descritos permitem determinar o poder calorífico superior de amostras de alimentos sólidos com uma precisão aceitável. Os valores energéticos das amostras de cereal de pequenoalmoço e de leite gordo em pó obtidos neste trabalho estão, de modo geral, de acordo com os valores apresentados nos respectivos rótulos das embalagens, os quais foram provavelmente estimados utilizando termos energéticos baseados na sua composição nutricional (uma prática corrente no meio industrial).

Deste modo, aplicando uma técnica calorimétrica fácil de implementar laboratorialmente, os estudantes de cursos na área da nutrição podem testar a aplicabilidade da Regra 4-9-4 para a estimativa do valor energético dos alimentos, comparando estes valores com os obtidos através de um método experimental.

\section{AGRADECIMENTOS}

Aos Profs. J. A. Martinho Simões (FC-UL), M. E. Minas da Piedade (FC-UL) e H. P. Diogo (IST-UTL) pela troca de ideias sobre a realização experimental dos ensaios de combustão e a escrita deste artigo. Este trabalho foi desenvolvido no Laboratório de Nutrição da Escola Superior Agrária de Santarém, Instituto Politécnico de Santarém.

\section{REFERÊNCIAS E NOTAS}

1. Atwater, W. O.; Woods, C. D.; The Chemical Composition of American Food Materials, U. S. Department of Agriculture; Office of Experiment Stations; Bulletin n. ${ }^{\circ} 28,1896$.

2. Merrill, A. L.; Watt, B. K.; Energy Value of Foods: Basis and Derivation, revised; U. S. Department of Agriculture; Agriculture Handbook n. ${ }^{\circ}$ 74, 1973.

3. Livesey, G.; Buss, D.; Coussement, P.; Edwards, D. G.; Howlett, J.; Jonas, D. A.; Kleiner, J. E.; Müller, D.; Sentko, A.; Food Control 2000, 11, 249.

4. Livesey, G.; Br. J. Nutr. 2001, 85, 271.

5. Warwick, P. M.; Baines, J.; Br. J. Nutr. 2000, 84, 897.

6. A energia metabolizável corresponde ao valor energético dos alimentos fisiologicamente disponível, isto é, à fracção de energia que fica disponível para o organismo como fonte de energia a partir dos alimentos ingeridos, depois de descontada a energia associada às fezes, urina e gases. ${ }^{4}$

7. U.S. Department of Agriculture, Agricultural Research Service; USDA National Nutrient Database for Standard Reference, Release 20, 2007. (Nutrient Data Laboratory Home Page, http://www.ars.usda.gov/ba/ bhnrc/ndl).

8. Food and Drug Administration; Federal Register 2003, 53, 2175.

9. European Communities; Official Journal of the European Communities 1990, $L 276,40$.

10. European Communities; Official Journal of the European Communities 2003, $L 333,51$.

11. ISO 1928:1995 (E); Solid Mineral Fuels - Determination of Gross Calorific Value by the Bomb Calorimetric Method, and Calculation of Net Calorific Value.
12. O processo de cálculo do coeficiente de digestibilidade de um alimento, D, descrito por Cohen, B. L.; Schilken, C. A.; J. Chem. Ed. 1994, 71, 342, baseia-se na utilização da expressão: $D=F_{1} \cdot D_{1}+$ $F_{\mathrm{g}} \cdot D_{\mathrm{g}}+F_{\mathrm{p}} . D_{\mathrm{p}}$, onde $D_{1,} D_{\mathrm{g}}$ e $D_{\mathrm{p}}$ são os coeficientes de digestibilidade dos lípidos, glícidos e proteínas, respectivamente, compilados para cada tipo de alimento ${ }^{2}$ e $F_{1}, F_{\mathrm{g}}$ e $F_{\mathrm{p}}$ são as fracções energéticas dos anteriores nutrientes no alimento em estudo. Os valores $F$ são calculados através da razão entre a energia de combustão produzida pelo nutriente $\left(m_{\mathrm{i}} \cdot Q_{\mathrm{c}, \mathrm{i}}\right)$ e a energia total de todos os nutrientes $\left(\Sigma m_{\mathrm{i}} \cdot Q_{\mathrm{c}, \mathrm{i}}\right)$, onde $Q_{\mathrm{c}, \mathrm{i}}$ são as energias mássicas de combustão de cada um dos referidos nutrientes para o tipo de alimento em estudo. ${ }^{2}$ Exemplificando para o caso do cereal utilizado neste trabalho: da Tabela 1 temos que $m_{1}=0,8 \mathrm{~g}, m_{g}=85 \mathrm{~g}$, e $m_{p}=6 \mathrm{~g}$; consultando a literatura ${ }^{2}$ para este tipo de alimento, obtém-se que $Q_{\mathrm{c}, 1}=9,30 \mathrm{kcal} \cdot \mathrm{g}^{-1}, Q_{\mathrm{c}, \mathrm{g}}=4,20 \mathrm{kcal} \cdot \mathrm{g}^{-1}$, $Q_{\text {c,p }}=5,80 \mathrm{kcal} . \mathrm{g}^{-1}, D_{1}=0,900, D_{\mathrm{g}}=0,979$, e $D_{\mathrm{p}}=0,667$; aplicando a expressão dada vem que $D_{\text {cereal }}=0,95$.

13. Southgate, D. A. T.; Durnin, J. V. G. A.; Br. J. Nutr. 1970, 24, 517.

14. Livesey, G.; Elia, M.; Am. J. Clin. Nutr. 1988, 47, 608.

15. Marca registada por Kellogg Co.; Battle Creek, Michigan, U.S.A. (http:// www.kellogg.com).

16. Lamprecht, I. Em Handbook of Thermal Analysis and Calorimetry; Vol. 4 - From Macromolecules to Man; Kemp, R. B., ed.; Elsevier: Amsterdam, 1999, chapter 4.

17. Marca registada por Société dês Produits Nestlé S. A., Vevey, Suiça. (http://www.nestle.com).

18. Parr Instrument Company; Instructions for the 1241 Adiabatic Oxygen Bomb Calorimeter, Manual n. ${ }^{\circ}$ 160; Moline - Illinois, 1981.

19. Kemp, R. B. Em Handbook of Thermal Analysis and Calorimetry; Vol. 1 - Principles and Practice; Brown, M. E., ed.; Elsevier: Amsterdam, 1998, chapter 14

20. Martinho Simões, J. A.; Castanho, M. A. R. B.; Lampreia, I. M. S.; Santos, F. J. V.; Castro, C. A. N.; Norberto, M. F.; Pamplona, M. T.; Mira, L.; Meireles, M. M.; Guia do Laboratório de Química e Bioquímica; Lidel - Edições Técnicas Lda.: Lisboa, 2000, p. 81.

21. NP 872:1983 (Ed. 2); Alimentos para Animais. Determinação do Teor de Cinza Total.

22. NP 875:1994 (Ed. 3); Alimentos para Animais. Determinação da Humidade.

23. A adição de água ao interior da bomba permite que o ácido nítrico formado no processo de combustão, devido à presença de azoto atmosférico residual e/ou da amostra conter azoto na sua composição, não se perca quando da libertação dos gases finais da combustão, permitindo obter um estado final para o processo de combustão bem definido. A quantidade de ácido nítrico gerada é posteriormente determinada para que se proceda à correcção da energia envolvida nesta reacção.

24. Podem-se formar vários ácidos (principalmente sulfúrico, nítrico e clorídrico) a partir de óxidos não metálicos durante a combustão de matéria orgânica. Neste trabalho, assumiu-se que todo o ácido titulado era ácido nítrico, porque a quantidade de ácido sulfúrico formado deverá ser desprezável, atendendo ao teor residual de enxofre detectado nas análises elementares. A quantidade de ácido clorídrico deverá também ser residual, dado as amostras não apresentarem teor em cloro quantificável.

25. Hubbard, W. N.; Scott, D. W.; Waddington, G. Em Experimental Thermochemistry; Vol. 1; Rossini, F. D., ed.; Interscience: New York, 1956, chapter 5 . 